\title{
El teléfono celular: una estrategia didáctica para la enseñanza del electromagnetismo
}

\author{
Domingo Padilla Arzúzar \\ Isabel Garzón Barragán ${ }^{1}$
}

Artículo recibido el 07 de mayo de 2008 y aprobado el 27de diciembre de 2008.

The cell phone a didactic strategy for

the teaching of electromagnetism

Resumen: Las ondas electromagnéticas, OEM, son una temática común en los currículos en las carreras de física e ingenierías. A pesar de ello, la manipulación de artefactos que conduce a que los estudiantes se formen una idea de los efectos de las OEM es escasa, en gran medida por la falta de equipos de laboratorio adecuados. Debido a esta problemática, nuestra investigación nos condujo a utilizar el teléfono celular, Tc, como instrumento de registro de las OEM. Junto con los estudiantes, logramos construir un blindaje electromagnético para el Tc y un reflector parabólico, en cuyo foco ubicamos un teléfono con conexión con el PC por el puerto USB para registrar las variaciones de la intensidad de la señal del operador celular.

Palabras clave: Ondas electromagnéticas, enseñanza del electromagnetismo, experimento con el teléfono celular.
Abstract: Electromagnetic waves is a subject included in most of the physics and engineering curricula, however, the handling of devises to allow students to become familiar with electromagnetic waves effects is scarce; mostly due to a lack of adequate lab equipment. Our research leads us to use a cellular phone as a recording instrument. With the students, we built an electromagnetic shield for the cellular phone and a parabolic reflector in whose focus we place a cellular phone connected to a PC via a USB port in order to register the changes in the operator's signal.

Key words: Electromagnetic waves, electromagnetism teaching, the mobile phone as a device for experimentation.

1 Universidad Pedagógica Nacional. Correo electrónico: igarzon@pedagogica.edu.co 


\section{Introducción}

Durante 1888 y 1889, Heinrich Hertz, profesor de la Universidad de Bonn, realizó una serie de experimentos que lo condujo a probar la existencia de las ondas electromagnéticas, predichas por Maxwell en sus trabajos sobre electrodinámica (Hertz, 1982). Desde entonces, los artefactos que funcionan con estos principios han tenido un desarrollo inconmensurable. Hoy día, por ejemplo, estamos invadidos de teléfonos celulares, redes de transmisión de datos inalámbricas, televisión satelital, etc. Todo gracias, en gran parte, a los descubrimientos de Hertz. Pero como suele pasar con los artefactos tecnológicos, lo que prima al final son las necesidades que suplen y las nuevas que crean y no los equipos como tal. En el caso del teléfono celular, la idea inicial fue suplir la necesidad de comunicarse con cualquier persona en cualquier momento y en cualquier lugar; de modo que el entendimiento, los estudios del funcionamiento de estos aparatos y las teorías físicas de fondo en su construcción quedan relegadas a un reducido grupo de personas (estos temas son abordados por los estudiantes de ciencias e ingenierías, cuyo número es bastante reducido en comparación con la población de un país).

Generalmente, cuando se menciona la frase teléfono celular en las aulas de clase es para pedir el favor de apagar o poner en modo silencioso estos equipos, pues resultan incómodos debido a las interrupciones abruptas, siendo fiel a su idea primigenia: en cualquier lugar y en cualquier momento. Casi nunca se utilizan de manera explícita para estudiar su funcionamiento. Paradójicamente, todos reconocemos que éstos funcio- nan, debido sobre todo, a las ondas electromagnéticas. Tan así que, cuando el aparato indica que la señal está baja, realizamos toda clase de maromas para que el teléfono la capte mejor: subirnos en lugares altos, salir a la ventana, retirarnos de los radios y televisores, etc. Con estas acciones estamos "teniendo en cuenta implícitamente los fenómenos de reflexión, refracción e interferencia de las ondas electromagnéticas"; a pesar de ello, cuando se estudian las ondas electromagnéticas en las clases de física en la escuela no se utiliza el teléfono celular como instrumento de registro de dichos fenómenos físicos, al contrario, preferimos mandarlos a apagar.

Ahora, cuando logramos abordar la temática de las ondas electromagnéticas, muy pocas instituciones educativas en Bogotá cuentan con equipos de ondas decimétricas y microondas (información tomada del inventario de instrumentos de laboratorio de ciencias naturales, realizado para el curso de capacitación de profesores de ciencias naturales del Distrito, ofrecido por la Universidad Pedagógica Nacional con el auspicio de la Alcaldía Distrital de Bogotá, entre febrero y diciembre de 2005), pues generalmente en las clases no se llega a esos temas y, viceversa, no se aborda el tema porque no existen los instrumentos de laboratorio que además son costosos. Nuestra propuesta gira en torno a la utilización del teléfono celular como instrumento de registro de las ondas electromagnéticas, pues creemos que al hacerlo de esta manera resulta significativo para los estudiantes de los cursos de física de media vocacional y algunos cursos en la universidad. 


\section{Acerca de la enseñanza de las ondas electromagnéticas}

La temática de las ondas electromagnéticas, овм, es abordada en el marco de los cursos de electromagnetismo o electrodinámica en las carreras de física y algunas ingenierías. En el caso del Departamento de Física de la Universidad Pedagógica Nacional, previo al curso de Electromagnetismo II, existe un curso de Mecánica de Ondas, situación que utilizamos a nuestro favor para introducir las actividades que se presentarán a continuación en este mismo documento.

En la mayoría de textos universitarios de electromagnetismo, las OЕM son abordadas desde el formalismo de las ecuaciones de Maxwell; es corriente encontrar los procedimientos matemáticos para llegar a la ecuación de onda desde las cuatro ecuaciones de Maxwell. Podríamos decir que se realiza una presentación de tipo operativo de las oEm. Para algunos estudiantes, este tipo de presentación resulta beneficiosa, pues condensa en pocas ecuaciones la esencia de la teoría electromagnética de campos. Sin embargo para los estudiantes novatos de ingeniería y ciencias físicas el asunto es a otro precio. Generalmente a estas alturas de la carrera aún no tienen bases sólidas en cálculo diferencial, integral, vectorial y tensorial. A duras penas están cimentando los conceptos fundamentales de estas matemáticas. Debido a estas problemáticas, nuestra propuesta se encamina a abrir los espacios en el aula para que los estudiantes tengan la oportunidad de construir sus experiencias básicas en torno a los efectos de las овм, en este caso, con un teléfono celular, Tc, pues, al igual que la familiarización con los efectos mecánicos, facilita el aprendizaje de la mecánica clásica.
Creemos que la familiarización con los efectos electromagnéticos facilitará el aprendizaje de la teoría electromagnética de campos (Trumper 2003, Sichau 2000 y Abrahams y Millar 2008).

Esta afirmación parece obvia, pero en las prácticas educativas se observan situaciones diferentes. Algunos proponen que se debe enseñar el formalismo matemático y acotejar los resultados a ciertos datos experimentales (Guisasola 1996). El inconveniente de esta propuesta reside en el hecho de que los estudiantes, en los niveles más altos donde se ven esos cursos, aún no tienen las destrezas matemáticas para lograrlo. Entonces éstos se quedan en la primera etapa del formalismo matemático con consecuencias desafortunadas: los estudiantes terminan creyendo que la física es un cúmulo de fórmulas y reglas de cálculo, en otras palabras, se crean falsas imágenes de las teorías físicas (Galili 1995 y Guisasola et al., 2008). Además, cada teoría física involucra algunos conceptos físicos básicos, un formalismo matemático y un conjunto de reglas de correspondencia que conecta los conceptos físicos con los objetos matemáticos que los representan (Ballentine, 2000). Las reglas de correspondencia inicialmente son usadas para expresar un problema físico en términos matemáticos. Una vez, la versión matemática del problema es formulada, puede ser resuelto por técnicas puramente matemáticas sin necesidad de una interpretación física. La solución formal es entonces traducida en términos físicos por medio de las reglas de correspondencia (Ballentine, 2000). Algunas veces esta conexión de conceptos físicos con objetos matemáticos es bastante obvia, sin embargo, en el caso del electromagnetismo, sin una 
debida preparación, no resulta tan fácil traducir los resultados formales en términos físicos (Guisasola, 2002 y Martín y Solbes, 2001).

Otro de los posibles inconvenientes de presentar inicialmente las oEm con el formalismo matemático puede estar en seguir el orden de los contenidos establecidos en los libros de texto universitarios (Pocovi y Finley, 2003). En general, un libro muestra una teoría electromagnética depurada, presentada en la manera en que el autor ha organizado sus ideas acerca de la teoría. Este autor ha tenido experiencias vitales que el lector (profesor o estudiante) ni se imagina. Además, es prácticamente imposible reconstruir todos los pasos que ha dado el autor para escribir el libro (Crawford, 1998), así que una mejor alternativa sería dejar que los estudiantes construyeran sus propias experiencias de los efectos de las ondas electromagnéticas, OEM, y es justo esta idea la que guía nuestro trabajo. Queremos que los estudiantes le den un nuevo significado a la frase onda electromagnética, más allá de su utilidad para transmitir información, y para ello creemos fuertemente que la familiarización con los efectos de las mismas es vital para lograr una verdadera comprensión de la teoría electromagnética de campos. Con esto no queremos decir que la familiarización con los efectos de las oEm basta para comprender la teoría electromagnética de campos, pero sí sienta bases sólidas para un abordaje más formal de teoría.

\section{Blindaje electromagnético}

La idea que guió esta actividad no fue construir representaciones gráficas de la apariencia física de las oEm, sino construir experiencias básicas desde los efectos electromagnéticos de las mismas. Para ello utilizamos el teléfono celular, Tc, como instrumento de registro de las oEm. Este dispositivo electrónico presenta dos grandes ventajas. Primero, el тc es de uso masivo y un gran número de estudiantes cuentan con el servicio de telefonía celular, lo cual reduce los costos drásticamente, en contraste con los altos precios de los equipos de laboratorio de ondas decimétricas y microondas; y, segundo, en su construcción se debió utilizar la teoría electromagnética en su máxima expresión. Esto lo convierte en un recurso casi inagotable, pues en su funcionamiento podemos encontrar desde lo más elemental hasta lo más complejo de la teoría electromagnética.

Lo primero que hicimos junto con los estudiantes fue inhibir la señal de un celular, es decir, impedir que llegue al aparato la onda portadora del operador de celular y que del celular salgan ondas electromagnéticas hacia el operador. Para lograr este efecto de blindaje electromagnético, utilizamos materiales de bajo costo y que se pueden conseguir en la cocina de cualquier hogar. Primero, buscamos una lata o envase metálico, en este caso, latas vacías de leche en polvo. Introdujimos el Tc en el interior del recipiente y lo sellamos con papel aluminio como lo muestra la Figura 1.

Con otro тс se marcó al que estaba en el recipiente $y$, de acuerdo con el operador, pudimos escuchar que el teléfono estaba fuera de servicio o estaba fuera de cobertura, o simplemente se iba a buzón de mensajes. Cualquiera de estos avisos indicaba que el teléfono no podía recibir la llamada. 


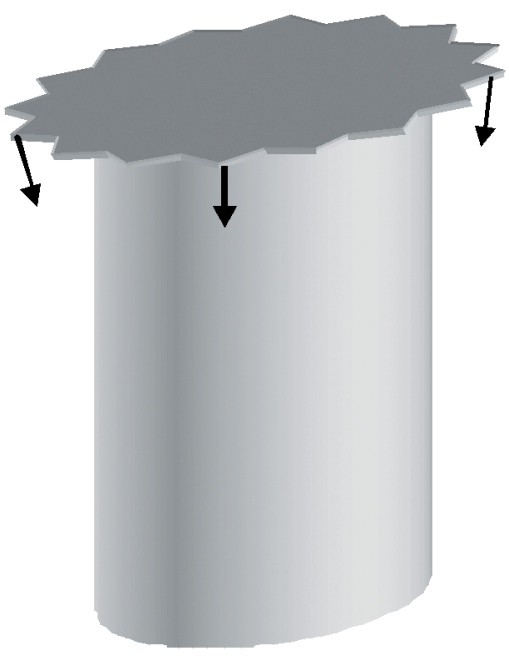

Figura 1: Blindaje electromagnético con tarro de leche en polvo y papel aluminio.

Luego quitamos la tapa de aluminio y en su lugar ubicamos una de plástico, nuevamente intentamos hacer la llamada al teléfono del recipiente, pero esta vez el teléfono sí pudo recibirla. De alguna manera, las ondas electromagnéticas del operador celular pudieron entrar y las ondas del teléfono pudieron salir del recipiente.

Entonces, nos preguntamos sobre las características de los materiales que se ubicaban alrededor del teléfono para inhibir la señal electromagnética. Pero como contábamos con imanes y multímetros, sólo pudimos determinar dos características consignadas en la Tabla 1.

En cursos anteriores, los estudiantes ya habían visto que el campo electrostático en el interior de una cavidad conductora cargada es nulo, lo cual indicaba que lo más probable era que en el caso electrodinámico de ahora, aun las características conductoras del material alrededor del Tc fueran las causantes de la inhibición de la señal del operador celular. Este supuesto se pudo corroborar rápidamente al observar la Tabla 2 en la cual claramente se ve que cuando el tc estaba rodeado de material conductor no había recepción de llamadas, como también se puede apreciar en la Figura 2.

\begin{tabular}{ll}
\hline \multicolumn{1}{c}{ Material } & \multicolumn{1}{c}{ Características } \\
\hline Envase de leche en polvo (latón). & Ferromagnético. Conductor de electricidad. \\
\hline Papel de aluminio. & No ferromagnético. Conductor de electricidad. \\
\hline Plástico para envolver. & No ferromagnético. Mal conductor de electricidad. \\
\hline
\end{tabular}

Tabla 1: Material para el blindaje.

\begin{tabular}{lc}
\hline \multicolumn{1}{c}{ Material } & Recepción de llamada \\
\hline Envase de leche en polvo (latón) tapado con papel aluminio. & No \\
\hline Envase de leche en polvo (latón) tapado con plástico. & Sí \\
\hline Envase de leche en polvo (latón) sin tapa. & Sí \\
\hline Tc envuelto en papel aluminio. & No \\
\hline
\end{tabular}

Tabla 2: Recipiente para el blindaje. 


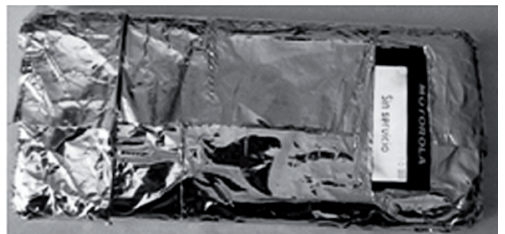

Figura 2: TC Motorola L6 envuelto en papel aluminio

Otra inquietud que surgió fue si necesariamente la cavidad conductora (lata de leche en polvo) tenía que estar totalmente cerrada (sin huecos). Entonces decidimos abrirles unos pequeños orificios por la tapa de papel aluminio, distribuidos en varias formas y tamaños, como lo muestra la Figura 3, complementada con la Tabla 3. Encontramos que la recepción de la llamada depende del diámetro de los orificios, la separación entre los mismos y el ángulo de la línea de huecos. Claramente los orificios actuaban como filtro.

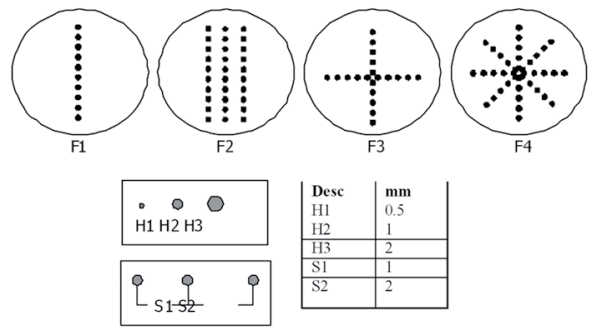

Figura 3: Configuración del blindaje. F: forma, $\mathrm{H}$ : diámetro de lo huecos, S: distancia entre huecos.

\begin{tabular}{ccc}
\hline NC & Detalles & Recepción de llamada \\
\hline 1 & $\mathrm{~F} 1, \mathrm{H} 1, \mathrm{~S} 1,0^{\circ}$ & No \\
\hline 2 & $\mathrm{~F} 1, \mathrm{H} 2, \mathrm{~S} 1,0^{\circ}$ & No \\
\hline 3 & $\mathrm{~F} 1, \mathrm{H} 3, \mathrm{~S} 1,0^{\circ}$ & Sí \\
\hline 4 & $\mathrm{~F} 2, \mathrm{H} 1, \mathrm{~S} 1,0^{\circ}$ & No \\
\hline 5 & $\mathrm{~F} 2, \mathrm{H} 2, \mathrm{~S} 1,0^{\circ}$ & No \\
\hline 6 & $\mathrm{~F} 2, \mathrm{H} 3, \mathrm{~S} 1,0^{\circ}$ & Sí \\
\hline 7 & $\mathrm{~F} 3, \mathrm{H} 1, \mathrm{~S} 1,0^{\circ}$ & No \\
\hline 9 & $\mathrm{~F} 4, \mathrm{H} 1, \mathrm{~S} 1,0^{\circ}$ & No \\
\hline 10 & $\mathrm{~F} 1, \mathrm{H} 1, \mathrm{~S} 2,0^{\circ}$ & No \\
\hline 11 & $\mathrm{~F} 1, \mathrm{H} 1, \mathrm{~S} 2,+90^{\circ}$ & No \\
\hline 12 & $\mathrm{~F} 2, \mathrm{H} 2, \mathrm{~S} 1,+90^{\circ}$ & Sí \\
\hline$\cdots$ & $\mathrm{F} 2, \mathrm{H} 2, \mathrm{~S} 1,-45^{\circ}$ & Sí, mala recepción. \\
\hline
\end{tabular}

Forma, diámetro de hueco, distancia entre huecos, giro (+ derecha, - izquierda).

Tabla 3: Configuración de la tapa del blindaje.

Uno de los grupos de estudiantes sugirió el siguiente razonamiento:

Si la información del teléfono celular viajara en forma de pequeñísimas partículas invisibles, hemos de esperar que se introduzcan por los orificios y se recibiera la llamada, pero los resultados no son esos, lo que se puede notar es que la forma en que se distribuyen los huecos juegan un papel importante, así como en el caso de las ondas en la cubeta de ondas.

Estas expresiones nos condujeron a reconocer que los estudiantes considera- 
ban la dependencia entre la longitud de onda (en el caso mecánico) y el tamaño de los obstáculos por donde éstas se propagan. Por ejemplo, si estuviéramos en un concierto de música al aire libre, frente a unos amplificadores potentes, $y$ nos fuéramos alejando poco a poco del tumulto de gente (obstáculos), podríamos notar que a medida que nos distanciamos de los parlantes escuchamos más los sonidos bajos que los agudos. Esto muestra claramente que los bajos (bajas frecuencias auditivas) se propagan con mayor facilidad por los obstáculos que los agudos (altas frecuencias auditivas), comportamiento característico de los fenómenos ondulatorios. Esta línea de razonamiento da nuevos elementos que contribuyen a entender las ondas electromagnéticas, sugiere una metodología experimental para determinar la frecuencia y la longitud de onda del TC y del operador celular. En la Figura 3, podemos observar las disposiciones de los agujeros en la tapa de aluminio del sistema de blindaje electromagnético, etiquetadas con la letra F, y en la Tabla 3 podemos encontrar algunas configuraciones utilizadas en las cuales es posible combinar disposición (F), diámetro de los agujeros (H), distancia entre huecos (S) y el ángulo de giro respecto a la posición inicial indicada en la Figura 3. Detallemos la configuración número 11 y 12 de la Tabla 3. En (11) la llamada entra sin problemas, pero en (12) el Tc timbra y luego se corta. La única diferencia entre estas dos configuraciones es el ángulo de disposición de la línea de huecos. Esto nos introdujo superficialmente al tema de polarización y se convirtió, por supuesto, en una nueva evidencia experimental para tratar de entender las
OEM, aunque no fue muy explorada por razones técnicas.

\section{Reflector parabólico}

Hay lugares donde la señal de los operadores celulares no "entra" o simplemente es muy tenue para realizar una llamada exitosa. Supusimos que un reflector parabólico sería útil para mejorar la señal, concentrando la mayor cantidad de radiación alrededor del teléfono. Para ello, aprovechando los diseños de las cocinas parabólicas solares (http:// solarcooking.org/espanol/cocina\%20 parab\%C3\%B3lica\%20plegable.pdf) con las que se concentra gran cantidad de luz solar en el foco de la parábola para cocer los alimentos, también fue posible utilizar un montaje parecido para concentrar la radiación de las frecuencias en que operan los teléfonos celulares.

Para la construcción del reflector parabólico utilizamos cartón, papel aluminio, grapadora, escuadra y un bisturí para cortar. Su interior se forró con aluminio para garantizar la reflexión de las ondas electromagnéticas. En la Figura 4 se encuentran el diseño y las dimensiones de una de las partes del reflector, este diagrama lo debemos trazar sobre el cartón que se quiere cortar; para ello, trazamos un línea o eje un tanto más largo que la suma de los segmentos d y e de la Figura 5 con ayuda de la escuadra trazamos, como lo muestra la Figura 5, la línea a perpendicular al eje, la misma operación se hace con las líneas b y c, teniendo en cuenta que los ángulos formados por las líneas a, b y c con el eje deben ser de $90^{\circ}$ (ángulos rectos). Con la ayuda de un bisturí, cortamos el primer cartón que va a servir de molde para el resto de las piezas, que en total deben sumar doce. 


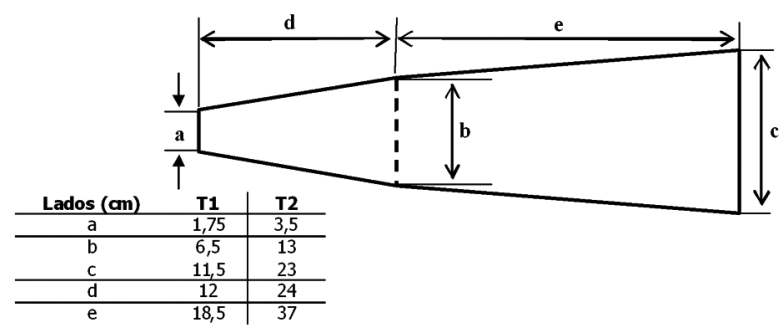

Figura 4: Dimensiones de la pieza del reflector.

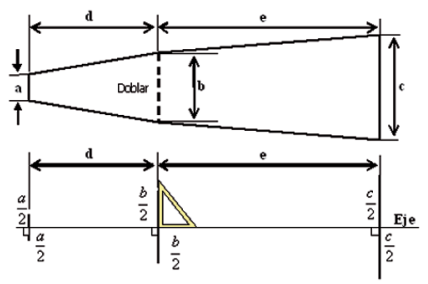

Figura 5: Diseño de una de las piezas del reflector.

Luego de cortadas todas las piezas de cartón, procedimos a forrarlas con papel aluminio. En la Figura 6, podemos ver la manera en que se adhiere el aluminio al cartón.

Luego de tener todas las piezas de cartón forradas con aluminio, procedimos a unirlas entre sí, como nos lo muestra la Figura 7. Las bisagras pueden ser cintas adhesivas o esparadrapos para aumentar el ajuste entre las piezas podemos coserlas con la grapadora de la misma manera en que se juntan las piezas 6 y 7 de la Figura 7.
Fuente: http://solarcooking.org con la debida autorización del autor para su publicación.

Al unir las piezas de cartón, nos quedó un hueco que llamamos tronera por donde introdujimos la última pieza (yugo), la cual se muestra en la Figura 8. El yugo consiste en dos anillos fabricados de tres capas pegadas de cartón, el diámetro de los anillos coincide con el diámetro exterior de un tubo de cartón en este caso obtuvimos uno del rollo de los paños de cocina. Los anillos también podrían construirse de madera, pero la perforación sería más dispendiosa sin herramientas adecuadas. Las pestañas interiores del reflector deben quedar aprisionadas entre los dos anillos, ajustada con los tornillos pasadores. El tubo de cartón no debe quedar muy ajustado, pues debe entrar y salir de los anillos para ajustar la posición del tc que va justo en el extremo que queda dentro del reflector. El resultado final lo podemos apreciar en la Figura 9 (A).

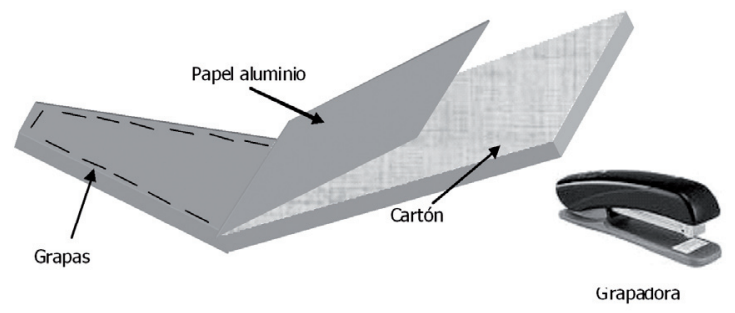

Figura 6: Forrado de aluminio de una de las piezas. 


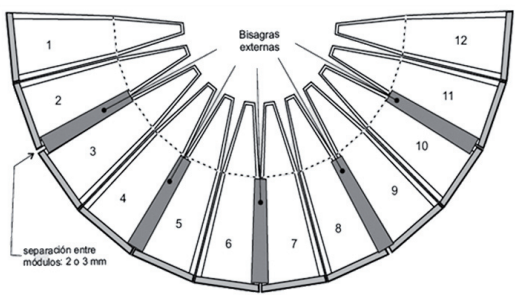

Figura 7: Disposición de las piezas de cartón del reflector.

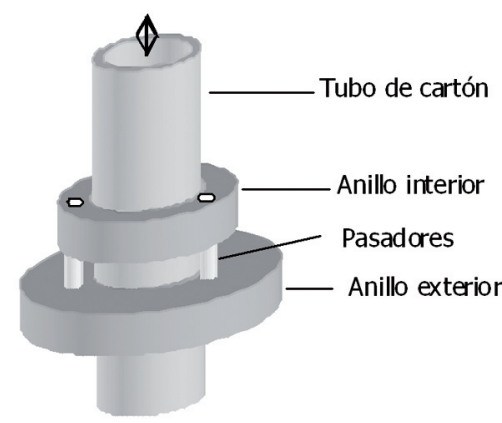

Figura 8: Yugo. Esta pieza ajusta el reflector y proporciona la base para el Tc.

Para medir la intensidad de la señal, utilizamos el Tc Motorola L6 con conexión por el puerto usB al PC y el software Phone Tool, proporcionado por el fabricante del Tc.
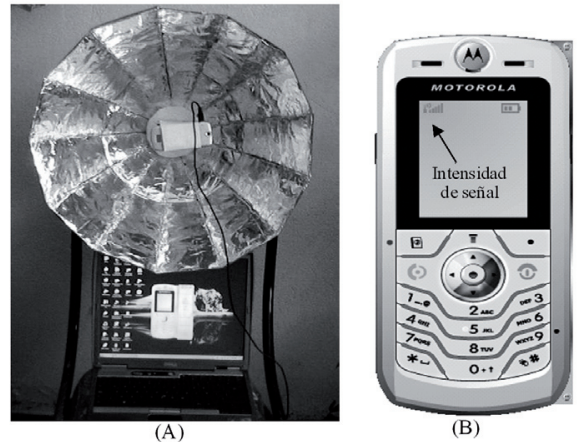

Figura 9: (A) Montaje final del reflector parabólico. Es capaz de aumentar la señal hasta un $40 \%$. (B) Software Phone Tool para el Tc Motorola L6.
Los resultados fueron sorprendentes: en un sitio donde la intensidad de la señal sólo alcanzaba el 29\% y el 48\%, con ayuda del reflector alcanzó un increíble $87 \%$, lo que mostró por una parte, la eficiencia del equipo construido y por otra, que definitivamente, las ozm sí se reflejan, a tal punto que podemos concentrarlas en una pequeña región del espacio.

\section{Conclusiones}

Quizás uno de los mejores resultados que podríamos haber tenido fue la posibilidad de vislumbrar nuevos caminos metodológicos para abordar las problemáticas de las oEm en los cursos de electromagnetismo del Departamento de Física de la Universidad Pedagógica Nacional, pues la carencia de materiales de laboratorio para realizar estas prácticas no permitía explorar más allá de la información proporcionada por el profesor acerca del funcionamiento de los artefactos. En esta ocasión, los estudiantes sí tuvieron la oportunidad de "experienciar" algunos efectos de las ondas electromagnéticas, particularmente en el Tc, desencadenándose una cascada de cuestionamientos interesantes que podrían ser tema de un próximo escrito.

Los estudiantes lograron identificar los efectos que tiene el blindaje de materiales conductores alrededor de los Tc, estos efectos posibilitaron una mejor 
compresión del comportamiento de las ondas electromagnéticas. También posibilitó que los estudiantes se cuestionaran acerca de las formas de propagación de las OEM, por los mecanismos de propagación de las ondas y cómo éstas permiten la comunicación.

Desde ese momento, se creó una nueva relación por parte de todos los miembros del equipo (profesores y estudiante) con los тc dentro y fuera del aula de clases. Ahora vemos estos

\section{Referencias bibliográficas}

Abrahams, I. y Millar, R. (2008). ¿Does practical work really work? A study of the effectiveness of practical work as a teaching and learning method in school science. International Journal of Science Education, 30(14), 19451969.

Ballentine, L. (2000). Quantum mechanics: a modern development. Londres: World Scientific Publishing.

Crawford, E. (1998). Michael Faraday on the learning of science and attitudes of mind. Science \& Education, 7(2), 203-211.

Galili, I. (1995). Mechanics background influences students' conceptions in electromagnetism. International Journal of Science Education, 17(3), 371-387.

Guisasola, J. (1996). Análisis crítico de la enseñanza de la electrostática en el bachillerato y propuesta alternativa de orientación constructivista. España: Universidad del País Vasco.

Guisasola, J., Zubimendi, J. L., Almudí, J. M. y Ceberio, M. (2002). The evolution of the concept of capacitance throughout the development of the electric theory and the understanding of its meaning by university students. Science \& Education, 11(3), 247-261. aparatos como instrumentos didácticos de fácil adquisición y, relativamente, de bajo costo, comparado con los equipos especializados de ondas decimétricas. Además, tenemos que recordar que nuestros estudiantes se están formando como Licenciados en Física, y es altamente probable que vayan a los colegios a enseñar esta disciplina, por lo cual creemos que esta experiencia contribuyó a aportar nuevas metodologías indirectamente también en esos lugares.

Guisasola, J., Zubimendi, J. L., Almudí, J. M. y Ceberio, M. (2008). Dificultades persistentes en el aprendizaje de la electricidad: estrategias de razonamiento de los estudiantes al explicar fenómenos de carga eléctrica. Enseñanza de las Ciencias, 26(2), 177-191.

Guisasola, J., Montero, A. y Fernandez, M. (2008). La historia del concepto de fuerza electromotriz en circuitos eléctricos y la elección de indicadores de aprendizaje comprensivo. Revista Brasileira de Ensino de Física, 30(1), 1604.1-1604.8.

Hertz, H. (1982). Ondas electromagnéticas. Barcelona: Serie de publicaciones de la Universidad Autónoma.

Jackson, J. (1962). Classical electrodynamics. Nueva York: John Wiley and Sons.

Martín, J. y Solbes, J. (2001). Diseño y evaluación de una propuesta para la enseñanza del concepto de campo en Física. Enseñanza de las ciencias, 19(3), 393-404.

Pocovi, M. C. y Finley, F. N. (2003). Historical evolution of the field view and textbook accounts. Science \& Education, 12(4), 387-396.

Sichau, C. (2000). Practising helps: thermodynamics, history, and experiment. Science \& Education, 9(4), 389-398. 